\title{
Stalling chromophore maturation of the fluorescent protein Venus reveals the molecular basis of the final oxidation step
}

\author{
Husam Sabah Auhimab\#, Bella L. Grigorenkocd\#, Tessa Harris ${ }^{a}$, Igor V. Polyakov ${ }^{\text {cd }}$, \\ Colin Berrya , Gabriel dos Passos Gomes ${ }^{\mathrm{e}, \mathrm{f}}$, Igor V. Alabuging*${ }^{*}$, Pierre J. Rizkallah ${ }^{\mathrm{h}}$, \\ Alexander V. Nemukhin ${ }^{\text {cd* }}$, D. Dafydd Jones ${ }^{a *}$.
}

\# These authors contributed equally to the work

a School of Biosciences, Molecular Biosciences Division, Cardiff University, Cardiff, UK

${ }^{\mathrm{b}}$ Department of Biology, College of Science, University of Baghdad, Baghdad, Iraq.

${ }^{c}$ Chemistry Department, Lomonosov Moscow State University, Moscow, Russian Federation

${ }^{\mathrm{d}}$ Emanuel Institute of Biochemical Physics, Russian Academy of Sciences, Moscow, Russian Federation

e Department of Chemistry, University of Toronto, 80 St. George Street, Toronto, ON M5S $3 \mathrm{H} 6$, Canada

${ }^{\mathrm{f}}$ Department of Computer Science, University of Toronto, 214 College St., Toronto, Ontario M5T 3A1, Canada.

g Department of Chemistry and Biochemistry, Florida State University, Tallahassee, FI 32306, USA

${ }^{\mathrm{h}}$ School of Medicine, Cardiff University, CF14 4XN, UK.

\section{* Corresponding authors.}

D. Dafydd Jones, School of Biosciences, Sir Martin Evans Building, Cardiff University, Cardiff CF10 3AX, UK. Email: jonesdd@cardiff.ac.uk. Tel: +44 2920874290

Alexander V. Nemukhin, Chemistry Department, Lomonosov Moscow State University, Leninskie Gory, 1-3, Moscow, Russian Federation. E-mail: anem@lcc.chem.msu.ru. Tel: +7 4959391096

Pierre J. Rizkallah, Institute of Infection \& Immunology, Sir Geraint Evans Building, School of Medicine, Heath Campus, Cardiff, CF14 4XN. Email: RizkallahP@cardiff.ac.uk. Tel: +442920742248

Igor V. Alabugin, Department of Chemistry and Biochemistry, Florida State University, Tallahassee, FI 32306, USA. Email: alabugin@chem.fsu.edu. Tel: +1 8506445795 


\begin{abstract}
Fluorescent proteins (FPs) have revolutionised the life sciences but the mechanism of chromophore maturation is still not fully understood. Incorporation of a photoresponsive non-canonical amino acid within the chromophore stalls maturation of Venus, a yellow FP, at an intermediate stage; the crystal structure reveals the presence of $\mathrm{O}_{2}$ located above a dehydrated enolate imidazolone (I) ring, close to the strictly conserved Gly67 that occupies a twisted conformation. His148 adopts an "open" conformation, potentially allowing $\mathrm{O}_{2}$ access to the chromophore. Absorption spectroscopy supported by QM/MM simulations suggest that the first oxidation step involves formation of a hydroperoxyl intermediate in conjunction with dehydrogenation of the methylene bridge. A fully conjugated mature chromophore is formed through release of $\mathrm{H}_{2} \mathrm{O}_{2}$ upon irradiation of this intermediate, both in vitro and in vivo. The possibility of interrupting and photochemically restarting chromophore maturation, and the mechanistic insights opens up new approaches for engineering optically controlled fluorescent proteins.
\end{abstract}




\section{Introduction}

Fluorescent proteins (FPs) represent a unique family of proteins that emit light in the visible region of the spectrum without the requirement of any additional cofactor, 1-4 so enabling them to act as genetically encoded markers and sensors ${ }^{5,6}$. Formation of the chromophore occurs through the stepwise covalent arrangement of three contiguous residues Xaa-Tyr-Gly (where Xaa can be various amino acid residues) in the presence of molecular $\mathrm{O}_{2}$ (Figure 1a). In the case of Venus, these three residues are 65-Gly-Tyr-Gly-67 (Figure 1a). Venus is a yellow fluorescent derivative of the classical Aequorea victoria GFP protein ${ }^{7}$. Amongst the various mutations introduced to generate this yellow variant, two key changes are the introduction of glycine at residue 65 (from serine) and tyrosine at residue 203 (from threonine); Tyr203 pistacks with the chromophore causing a red shift in excitation and emission into yellow region ${ }^{7,8}$. The mature chromophore has an extended conjugated bond network comprised of the aromatic $\mathrm{P}$ ring and the imidazolone I ring linked via a methylene bridge (see Figure 1a for ring and atom notation), which is located within the core of the $\beta$-barrel structure ${ }^{1,4,9,10}$. The first step in chromophore formation is cyclisation through linkage of the backbone amine of the strictly conserved Gly67 residue to the carbonyl carbon of the Xaa 65 residue (Gly65 in the case of Venus). Cyclisation is followed by dehydration $/ \mathrm{O}_{2}$-dependent oxidation steps to generate a fully conjugated system. There has been much discussion about the exact mechanism of chromophore formation, especially the order of the dehydration and dehydrogenation/oxidation steps ${ }^{4,9,10}$, and the role $\mathrm{O}_{2}$ plays in the rate-limiting oxidation step ${ }^{11}$. The availably of structures representing trapped intermediates has helped shed light on potential mechanisms ${ }^{12-16}$ but there are still questions to be addresed. To our knowledge, neither the crucial $\mathrm{O}_{2}$ nor the oxygenated intermediates have been observed, while theoretical simulations ${ }^{17}$ have speculated on the mechanism of action. Gly67 is strictly conserved and critical to maturation 15,18 , but its role is unclear.

Manipulating the chemical properties of the chromophore by protein engineering, either directly through changes to two of the three chromophore residues, or indirectly through changing the chromophore environment, have generated a range of new fluorescent proteins, including Venus ${ }^{7}$, with properties suited to their 
particular application ${ }^{6}$. One of the most important FP class for super-resolution imaging is the photo-controllable FPs, whereby fluorescence is either switched on/off, or spectral properties significantly shifted in response to light ${ }^{19-22}$. Mechanisms of action involve chemical modifications such as decarboxylation of Glu222 (e.g. PA-GFP ${ }^{23}$ ), backbone cleavage (e.g. Kaede ${ }^{24}$ ) and chromophore hydration (e.g. Dreiklang ${ }^{25}$ ), or conformational changes such as chromophore cis/trans isomerisation (e.g. Dronpa ${ }^{26}$, rsGFP ${ }^{27}$ ). The use of photochemically active non-canonical amino acids (ncAA) has further expanded optical control approaches 28. Phenyl azide photochemistry is particularly useful as it has been used to turn on, off, or switch the fluorescence properties of green ${ }^{29,30}$ and red ${ }^{31}$ FP types.

Here we use the photochemical properties of genetically encoded phenyl azide to stall chromophore maturation of Venus at an immature non-fluorescent intermediate (termed im-Venus ${ }^{66 a z F}$ ) state before UV irradiation instigates maturation to a final fluorescent form. The structure of the intermediate reveals the protein has undergone the dehydration but not the oxidation step. Additional new structural features add further new insights, including an essential role for the strictly conserved Gly67 and, for the first time, experimental observation of molecular $\mathrm{O}_{2}$ in direct proximity to the immature chromophore. The combination of experimental spectroscopy with quantum mechanical/molecular mechanics (QM/MM) simulations allowed us to propose a mechanism for the $\mathrm{O}_{2}$ dependent oxidation step whereby a hydroperoxyl intermediate is formed as part of the oxidation mechanism. 

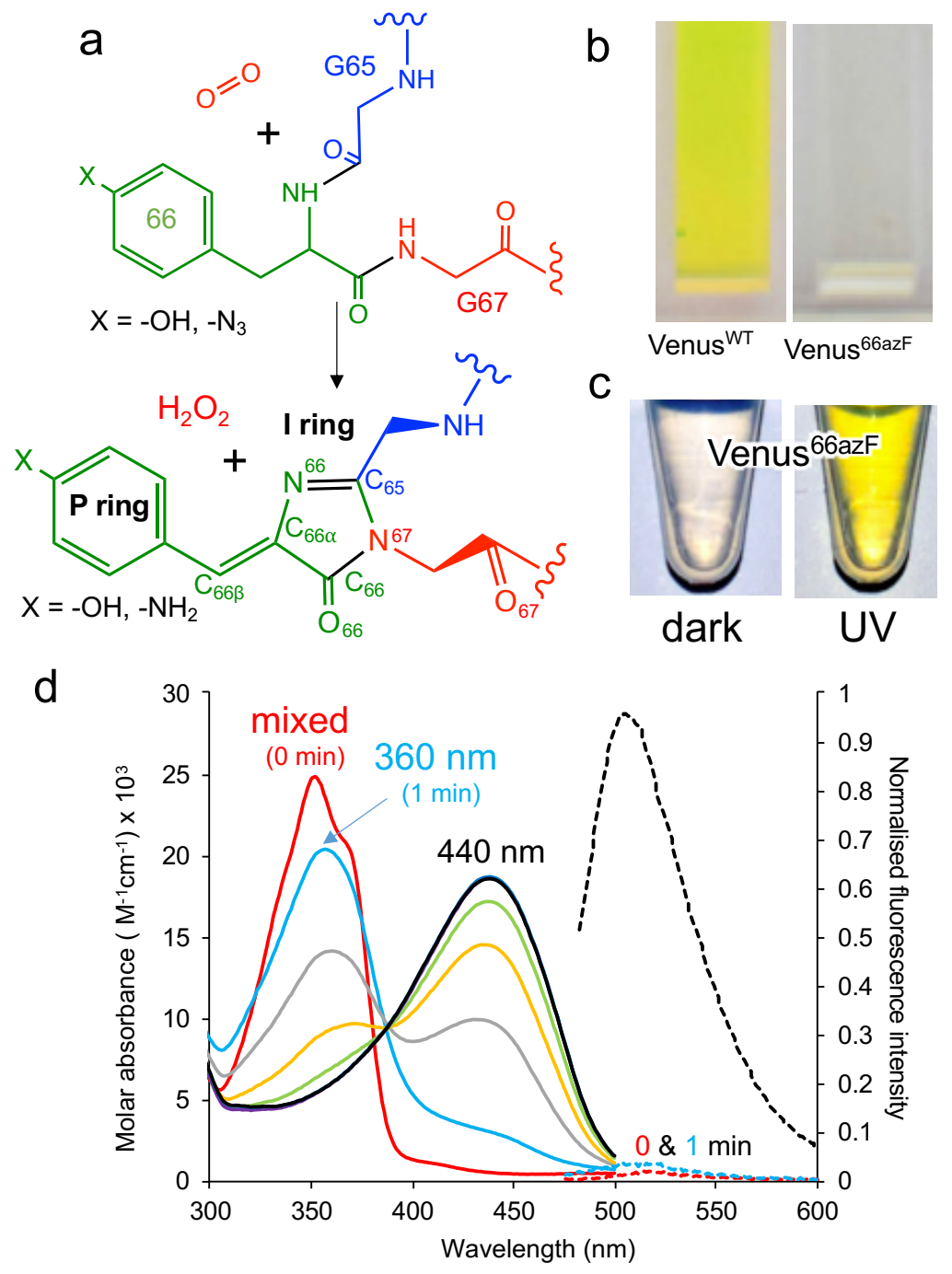

Figure 1. Effect of azF incorporation at residue 66 of Venus. (a) Scheme outlining the basic maturation of Venus. The bond and ring nomenclature are described in the lower structure. (b) Solution colours of Venus ${ }^{W T}$ and Venus ${ }^{66 a z F}$. (c) Solution colours of Venus ${ }^{66 a z F}$ before (dark) and after (UV) illumination with UV light. (d) Absorbance (solid line) and fluorescence (dashed line) of Venus ${ }^{66 a z F}$. Red, light blue, grey, orange, green, purple, dark blue represent spectra after $0,1,5,10,15$, 30, 45 and 60 min. Full spectral properties are shown in Supporting Table S1. Full fluorescence emission time course and in vivo imaging are shown in Supporting Figure S1.

\section{Results}

Incorporation of phenyl azide chemistry within the Venus chromophore 
To incorporate a photochemical switch into Venus, we replaced the chromophore residue Tyr66 with the aromatic non-canonical amino acid $p$-azido-L-phenylalanine $(\mathrm{azF})^{32,33}$; effectively the hydroxyl group of tyrosine is replaced with an azide group. The variant termed Venus ${ }^{66 a z F}$ is produced as a colourless protein (Figure $1 \mathrm{~b}$ ) with no inherent fluorescence (Figure $1 \mathrm{~b}-\mathrm{d}$ and $\mathrm{S} 1$ ), and acts an excellent starting point for an optically controlled FP. The absorbance spectrum of the colourless nonfluorescent Venus ${ }^{66 a z F}$ reveals the protein absorbs in the UV region. Absorbance peaks at $\sim 350 \mathrm{~nm}$ with a significant shoulder at $\sim 360-370 \mathrm{~nm}$; no fluorescence is observed from this species (Figure $1 \mathrm{~d}$ and S1a-b). Irradiation of Venus ${ }^{66 a z F}$ with near-UV light converts the protein to a coloured and fluorescent form both in vitro (Figure 1c-d, S1a-b) and in vivo (Figure S1c-d), with an absorbance $\lambda_{\max }$ shifting to 440 nm (Supporting Table S1). Fluorescence emission increased over 100-fold on activation, allowing imaging of bacterial cells expressing the activated Venus ${ }^{66 a z F}$ (Figure S1d). Spectroscopic analysis of the time course of activation reveals a potential two-step process. Within 1 min of UV exposure, the double hump absorbance spectrum feature disappears, with the remainder of the time points forming a clear isosbestic point at $\sim 390 \mathrm{~nm}$ suggesting conversion directly from one form to another. As the initial dark sample diverges away from the isosbestic point, it suggests a primary conversion to an intermediate species, which is also nonfluorescent, followed by a slower conversion to the activated form.

a

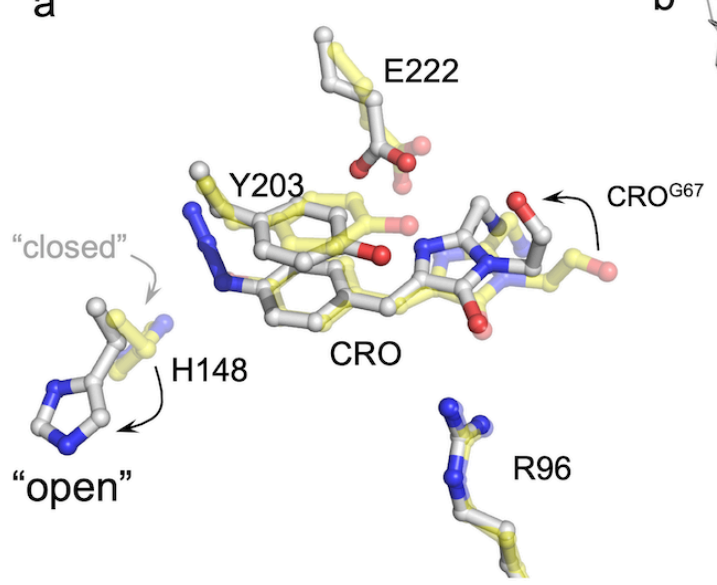

b

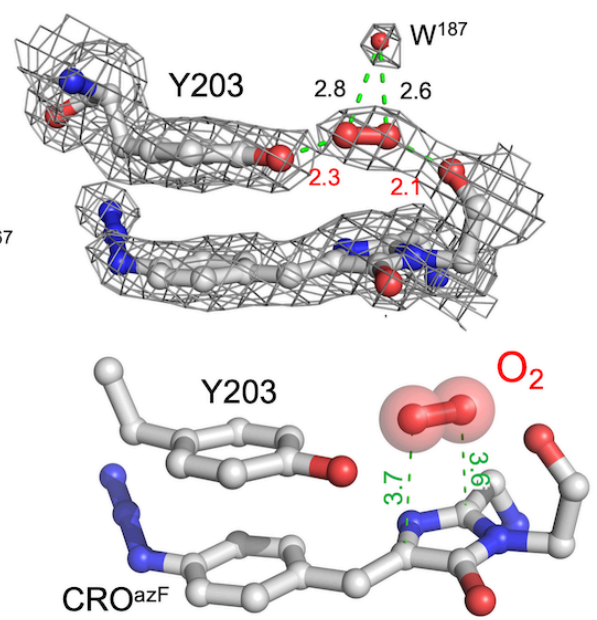

Figure 2. Structure of im-Venus ${ }^{66 a z F}$ proximal to the chromophore. (a) comparison of im-Venus ${ }^{66 a z F}$ (grey; PDB 6sm0) with Venus (yellow; PDB $1 \mathrm{mwy}^{8}$ ). $\mathrm{CRO}$ is the chromophore (Gly65-Tyr/AzF66 -Gly67). (b) Position of the $\mathrm{O}_{2}$ molecule 
in im-Venus ${ }^{66 a z F}$. The top panel shows the electron density (2Fo-Fc, 1.5 sigma) for the $\mathrm{CRO}, \mathrm{Y} 203$ and $\mathrm{O}_{2}$ together with an additional water molecule. The lower panel removes the electron density for clarity. Relevant distances are shown in $\AA$.

\section{Structure of im-Venus ${ }^{66 a z F}$}

The structure of the pre-photoactivated Venus ${ }^{66 a z F}$ (from herein referred to as immature or im-Venus ${ }^{66 a z F}$ ) was determined (see Supporting Table S2 for structural statistics) to gain an insight into the molecular nature of the non-fluorescent, immature chromophore. The general structure of im-Venus ${ }^{66 a z F}$ is similar to mature Venus (henceforth termed Venus ${ }^{\mathrm{WT}}$ ), with a root-mean-squared deviation (rmsd) over the backbone of $0.39 \AA$ (Supporting Figure S2a). The most significant differences occur in and around the chromophore (termed CRO). Electron density fits well to a cyclised chromophore containing the intact azide group present on the $P$ ring (Figure 2 and S2b). The lack of the electron density protruding from the I ring at the $\mathrm{C}_{65}$ position suggests the dehydration step in chromophore maturation has occurred by this point (Supporting Figure S2b). Compared to Venus ${ }^{W T}$, the I ring component shifts position in im-Venus ${ }^{66 a z F}$ due to the more acute angle in the methylene bridge (Figure S3b). A comparison of the residues surrounding the CRO shows that several residues exist in different conformations compared to Venus (Figure S3). His148, a critical $\mathrm{H}$-bonding residue to the mature $\mathrm{CRO}$, exists fully in the open-gate conformation together with Glu222, another residue necessary for function, and Tyr203 (pi-stacks with the CRO in Venus ${ }^{\mathrm{WT}}$ ) also shift position with respect to the CRO (Figure $2 a$ ).

Further analysis of the chromophore reveals several novel features that provide us with insights into $\mathrm{CRO}$ maturation and the role of molecular $\mathrm{O}_{2}$ in the process. In imVenus $^{66 a z F}$, the backbone carbonyl of the strictly conserved Gly67 is $\sim 180^{\circ}$ out of position compared to that observed in other FPs (Figure 2a-b). The C-O bond is also longer $(1.36 \AA)$ than that generally observed for a carbonyl $\mathrm{C}-\mathrm{O}$ bond within the context of a peptide bond $(\sim 1.24 \AA)$. To our knowledge, the only other time this conformation has been observed is in the unpublished structure of an immature chromophore of a GFP maturation disabling mutant determined by the Getzoff group (PDB 2qt2; Supporting Figure S4). There is additional electron density sandwiched between the Gly67 carbonyl group and the hydroxyl group of Tyr203 (Figure 2b), 
which we have assigned to molecular $\mathrm{O}_{2}$ after attempting to: (1) refine the structure with two $\mathrm{H}_{2} \mathrm{O}$ but the presence of Tyr203 effectively blocks the ability of two water molecules to occupy this position; (2) refinement of im-Venus ${ }^{66 a z F}$ with one water molecule left an elongated tail of density; (3) molecular $\mathrm{O}_{2}$ fitted best. The $\mathrm{O}_{2}$ molecule lies between the carbonyl oxygen of Gly67 and the hydroxyl group of Tyr203 above the plane of I ring element (Figure $2 b$ ). $\mathrm{O}_{2}$ has been postulated to be positioned either above the plane of the chromophore facing Glu222/Tyr203 or below the chromophore plane facing Arg96; here it is clear $\mathrm{O}_{2}$ above the plane of the chromophore on the Glu222/Tyr203 face (Figure 2b).
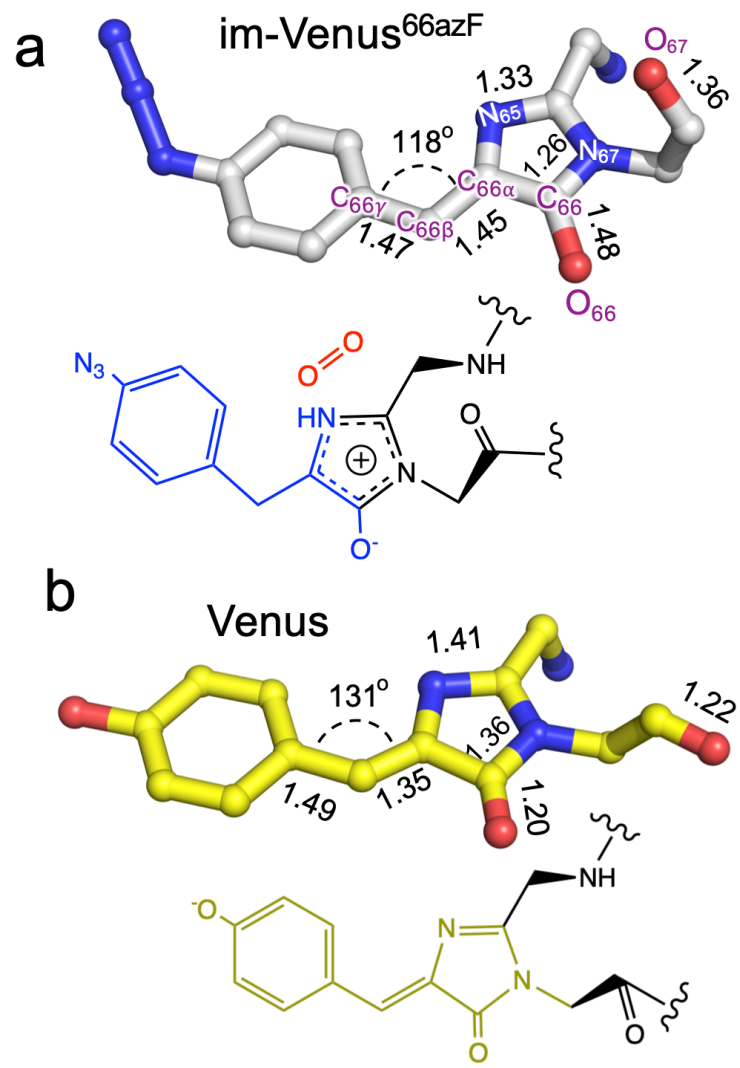

Figure 3. Chromophore bond distances and angles for im-Venus ${ }^{66 a z F}$ and mature Venus ${ }^{\mathrm{WT}}$ fluorescent proteins. (a) im-Venus ${ }^{66 a z \mathrm{~F}}$ with proposed chemical structure; (b) Venus ${ }^{W T}$ (PDB code $1 \mathrm{myw}^{8}$ ) with the chemical structure. A full list of bond lengths can be found in Supporting Table S3.

Bond lengths and angles also provide an insight into the structure of the trapped chromophore intermediate (Figure 3; Supporting Table 3). During maturation, $\mathrm{O}_{2}$ is 
thought to be involved in generating the final $\mathrm{C}=\mathrm{C}$ that links the P-ring and I ring $\left(\mathrm{C}_{66 \beta}-\mathrm{C}_{66 \alpha}\right.$ to $\left.\mathrm{C}_{66 \beta}=\mathrm{C}_{66 \alpha}\right)$. In Venus, the $\mathrm{C}_{66 \beta}-\mathrm{C}_{66 \alpha}$ bond is $1.35 \AA$, as expected for a $\mathrm{C}=\mathrm{C}$ while the same bond is $1.45 \AA$ im-Venus ${ }^{66 \mathrm{azF}}$, similar in length to adjacent $\mathrm{C}_{66 \gamma^{-}}$ $\mathrm{C}_{66 \beta}$ in both proteins (Figure 3a). This suggests that the $\mathrm{C}_{66 \beta}-\mathrm{C}_{66 \alpha}$ in im-Venus ${ }^{66 a z F}$ remains as a single bond. The bond angle between $\mathrm{C}_{66 \gamma}-\mathrm{C}_{66 \beta}-\mathrm{C}_{66 \alpha}$ is also more acute for im-Venus ${ }^{66 a z F}\left(118^{\circ}\right.$ versus $131^{\circ}$ for Venus; Figure 3 ). In the I-ring, the $\mathrm{C}_{66}-\mathrm{O}_{66}$ bond is $1.48 \AA$ for im-Venus ${ }^{66 a z F}$, which is longer than would be expected for a ketocarbonyl $\mathrm{C}=\mathrm{O}$ bond $(1.20 \AA$ ) as observed in Venus (Figure $3 \mathrm{~b}$ ) and a standard C-O single bond (1.43 $\AA$ ). This longer $\mathrm{C}-\mathrm{O}$ bond also makes polar contacts with the critical maturation Arg96, a residue that occupies a near-identical position in Venus $^{\text {WT }}$ (Figure 2a). Based on these measurements, we predict that the enolate is the most likely form of $\mathrm{C}_{66}-\mathrm{O}_{66}$ (Figure 3a), as has been proposed previously for the dehydrated intermediate ${ }^{16}$, with Arg96 stabilising the negative charge. The associated $\mathrm{C}_{66}-\mathrm{N}_{67}$ bond is shorter (1.26 $\AA$ ) compared to Venus (1.36 $\AA$ ). The $\mathrm{C}_{66}{ }^{-}$ $\mathrm{N}_{67}$ constrained within the cyclic configuration could indicate that this section of the ring is fixed as an ${ }^{-} \mathrm{O}-\mathrm{C}=\mathrm{N}$ arrangement. Analysis of the rest of the bond lengths comprising the I ring (Figure 3a and Table S3), together with the trigonal planar arrangement of the I ring with the $\mathrm{C}_{66 \beta}$, suggests that there may be a delocalised system around the I ring, with the positive charge spread around the ring. 


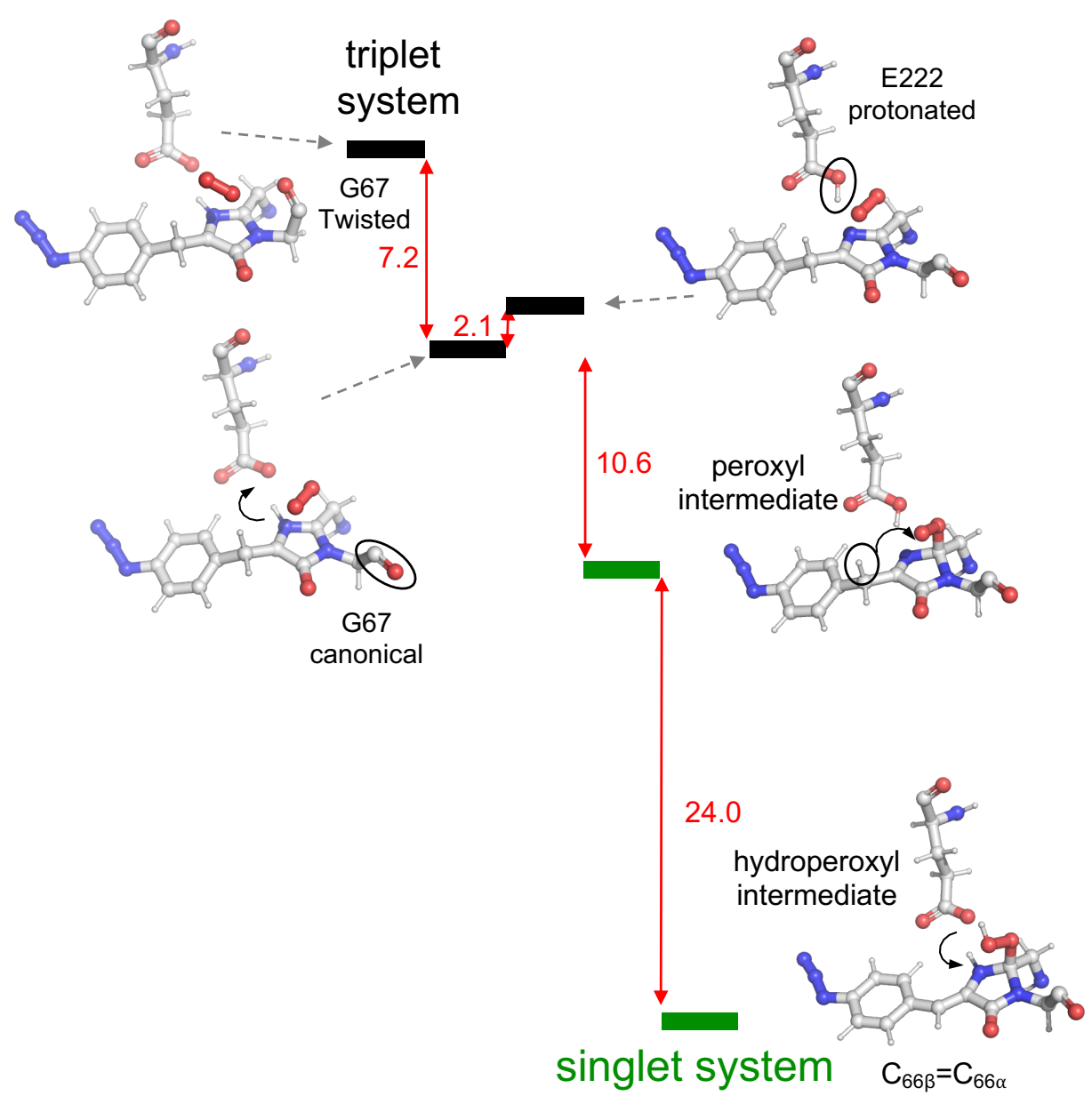

Figure 4. Reaction scheme for oxygenation of the chromophore as predicted by QM/MM modelling. Triplet and singlet systems are differentiated by black and green lines, as indicated in the diagram. The $\Delta \mathrm{E}$ between each state is shown in red with units of $\mathrm{kcal} / \mathrm{mol}$. Significant changes between each state are outlined in the diagram.

\section{Simulation of the oxidative step of chromophore maturation.}

To correlate the structural and functional observations, modelling of potential intermediates was performed using the atomic coordinates of im-Venus ${ }^{66 a z F}$ obtained in this work as a starting point and was associated with the observed absorbance data. The overall scheme based on the simulations is shown in Figure 4. The simulations predict that the first step is Gly67 switches to its energetically more favourable ( $7 \mathrm{kcal} / \mathrm{mol})$ canonical configuration. Triplet state oxygen can now access the I ring with concomitant protonation of Glu222, which acts as a general acid/base in the maturation scheme. The oxidation steps then proceed starting from partial negative charge transfer to $\mathrm{O}_{2}$, which switches from the triplet to singlet state. 
Glu222 is protonated with $\mathrm{N}_{65}$ donating the proton (Figure S5). The $\mathrm{O}_{2}$ then attacks $\mathrm{C}_{65}$ (and not $\mathrm{C}_{66 \alpha}$ ) generating a peroxy intermediate (Figures 4 and S5). The peroxy anion then abstracts a proton from $\mathrm{C}_{66 \beta}$ to form the stable hydroperoxyl intermediate with Glu222 protonating $\mathrm{N}_{65}$ (Figure 4 and S5); the hydroperoxyl species has a predicted absorbance of $360 \mathrm{~nm}$. As well as Gly67 converting through to its canonical conformation, the formation of the methylene bridge between the I and $\mathrm{P}$ rings results in a shift to a configuration similar to that observed for the mature chromophore observed in Venus ${ }^{\mathrm{WT}}$; the methylene bridge bond angle is now $134^{\circ}$ (Figure 5a).

The lone pair of the hydroperoxyl group $(-\mathrm{OOH})$ in the model has near-ideal alignment with the two $\mathrm{C}-\mathrm{N}$ bonds (Figure $5 \mathrm{c}-\mathrm{d}$ ). This favourable stereoelectronic arrangement activates stabilizing hyperconjugative $n_{\circ} \rightarrow \sigma^{*} \mathrm{CN}$ interactions, which can partially compensate for the loss of aromatic stabilisation in the I ring. This is complemented by two strong $n_{N} \rightarrow \sigma^{*}$ co interactions. The importance of the latter effect is expected to grow further in the transition state for the final C-O bond scission where it provides an important transition state stabilization effect that can significantly assist the final aromatizing step of the cascade. ${ }^{34-36}$

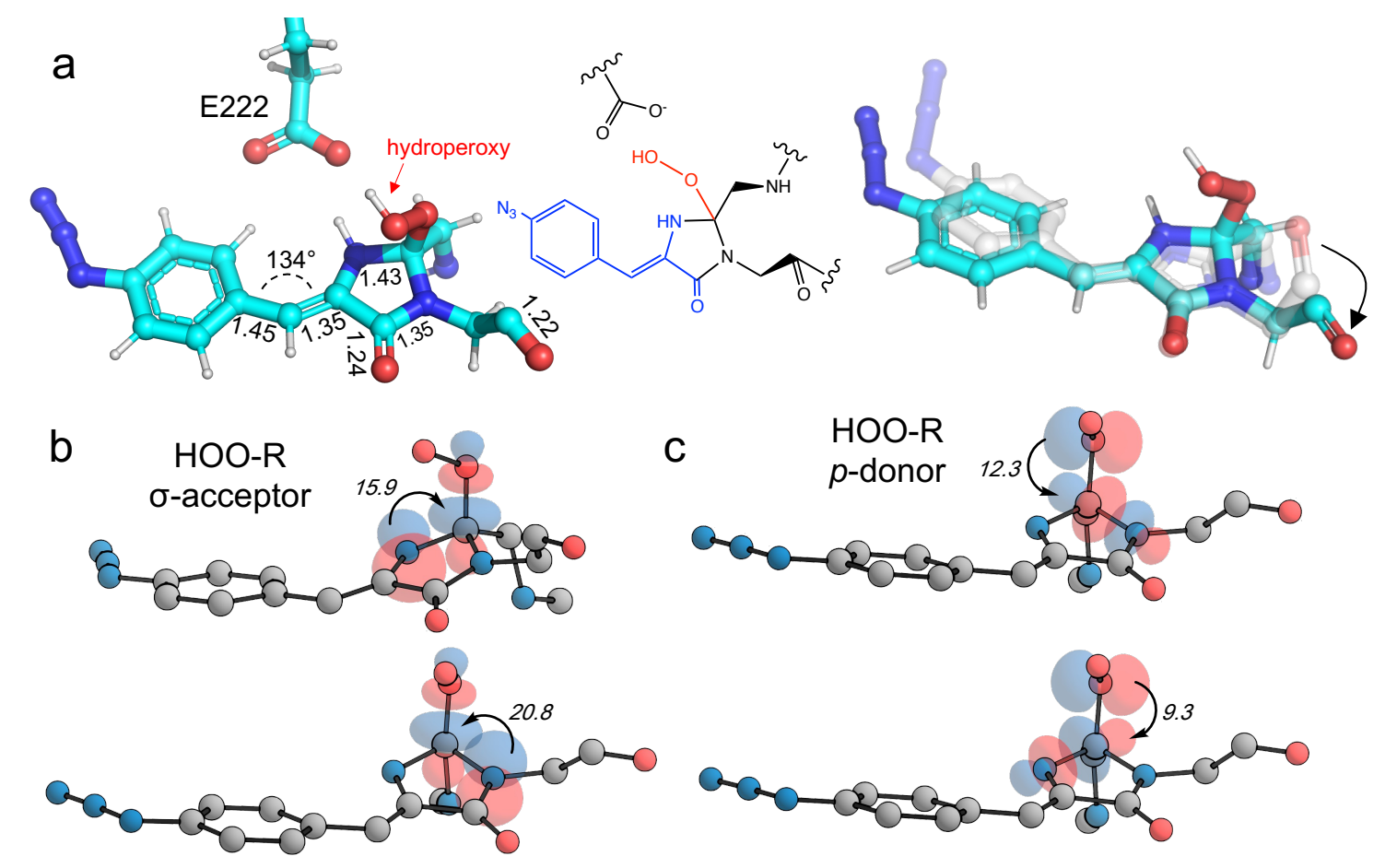


Figure 5. Models of the hydroperoxyl intermediate. (a) Model structure as predicted by $\mathrm{QM} / \mathrm{MM}$ simulations with the hydroperoxy group bound to $\mathrm{C}_{65}$ as indicated. The chemical structure of the intermediate is also shown. On the right-hand side is a comparison of the modelled hydroperoxyl intermediate (cyan) with the crystal structure of im-Venus ${ }^{66 a z F}$. (b-c) Stereoelectronic interactions in hydroperoxyl intermediate (hydrogen atoms omitted for clarity with the numbers related to energies in $\mathrm{kcal} / \mathrm{mol}$ ). Both the hydroperoxyl moiety acting as a $\sigma$-acceptor (b) and $p$ donor (c) are shown.

The final step occurring over the irradiation cycle is the conversion to a fully mature fluorescent chromophore. Two events need to be considered: reduction of the azide and full conjugation of the chromophore through a loss of the hydroperoxyl moiety. The spectral properties suggest that the final end product is likely to be the phenylamine form of the mature chromophore as has been observed before (Table $\mathrm{S} 1$ and ${ }^{30}$ ). Simulations concur with this with the final product having a predict absorbance max at $444 \mathrm{~nm}$, close to the $440 \mathrm{~nm}$ observed in Figure 1d. Full chromophore conjugation with the azide left intact will generate a species less stable than the preceding step and has a predicted absorbance maximum at $451 \mathrm{~nm}$ (Supporting Figure S6). The alternative route appears more likely: reduction to the phenylamine followed by loss of the hydroperoxyl group (generating $\mathrm{H}_{2} \mathrm{O}_{2}$ ). The phenylamine version of hydroperoxyl intermediate has a predicted absorbance maximum of $367 \mathrm{~nm}$. Based on the experimental time course observed in the Figure $1 \mathrm{~d}$, the initial species mix dominated by the hydroperoxyl intermediate (predicted $\lambda_{\max }=360 \mathrm{~nm}$ ) within $1 \mathrm{~min}$ of irradiation converts to the dominant hydroperoxyl phenylamine (predicted $\lambda_{\max }=367 \mathrm{~nm}$ ) that then forms the mature phenylamine chromophore.

\section{Oxygen access to the chromophore and the twisted Gly67}

The structure of im-Venus ${ }^{66 a z F}$ revealed the presence of $\mathrm{O}_{2}$ within the core of the protein close to the I ring of the chromophore. There are two potential ways in which $\mathrm{O}_{2}$ becomes located in such a position: during the folding process or diffusion into the core after folding and the cyclisation event. Simulations, whereby $\mathrm{O}_{2}$ is replaced by a water molecule, reveal that the twisted conformation of the immature 
chromophore is more stable by $18.1 \mathrm{kcal} / \mathrm{mol}$ than the canonical form (Figure 6a). The presence of water is unsurprising given that $\mathrm{O}_{2}$ is not required for the preceding cyclisation and dehydration events. Thus, water helps stabilise the twisted form of Gly67 present in im-Venus ${ }^{66 a z F}$. If this is the case, $\mathrm{O}_{2}$ is likely to displace the water molecule as part of the maturation mechanism, meaning it has to gain access to the core of the protein. Analysis of the im-Venus ${ }^{66 a z F}$ structure reveals that the "open" His148 conformation may play a role (Figure 6b-c). A channel leads to the chromophore in im-Venus ${ }^{66 a z F}$ that is only available if His 148 occupies the "open" state; when His148 occupies the "closed" state normally observed in mature fluorescent proteins, the tunnel becomes blocked.

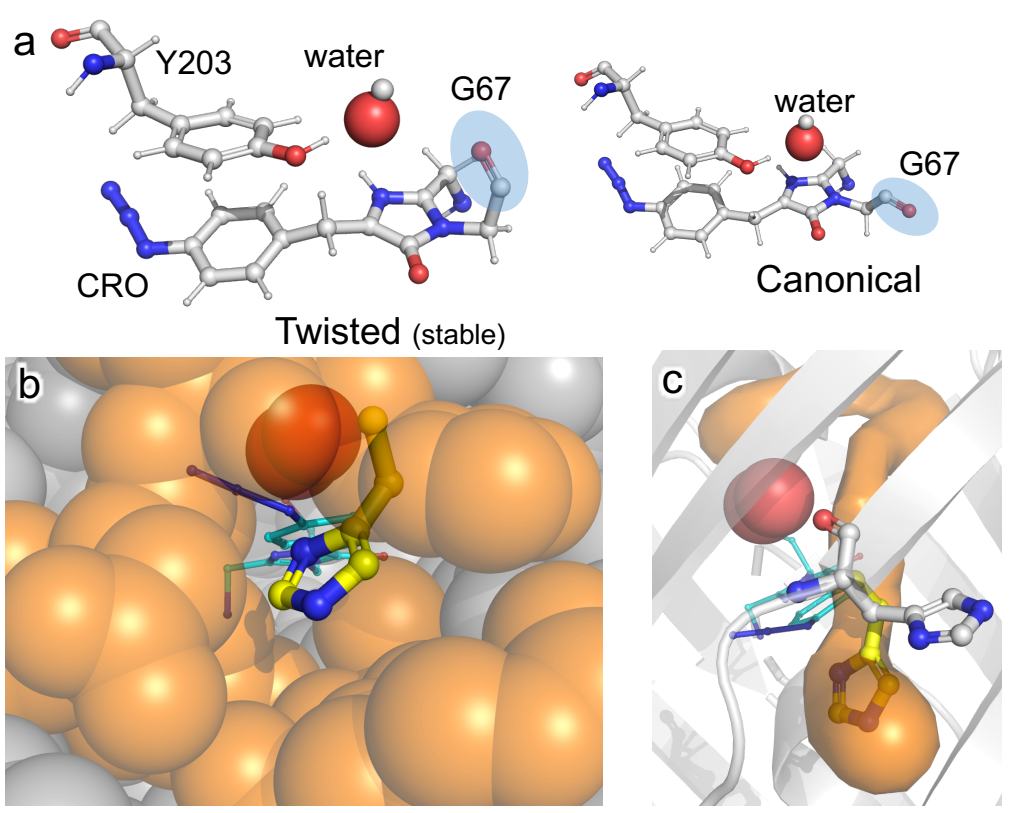

Figure 6. Role of water and internal tunnels in chromophore maturation. (a)

Simulation of the twisted conformation stabilised by a water molecule in place of $\mathrm{O}_{2}$ (and for reference, the canonical form usually observed in mature Venus ${ }^{W T}$ conformation). The twisted form is more stable by $18.1 \mathrm{kcal} / \mathrm{mol}$ compared to the canonical form. The water molecule of interest is highlighted. Chromophore accessibility, as shown by (b) spheres and (c) CAVER tunnel analysis ${ }^{37}$. The alternative conformation for His148 (yellow sticks) in Venus ${ }^{\mathrm{WT}}$ is shown. 


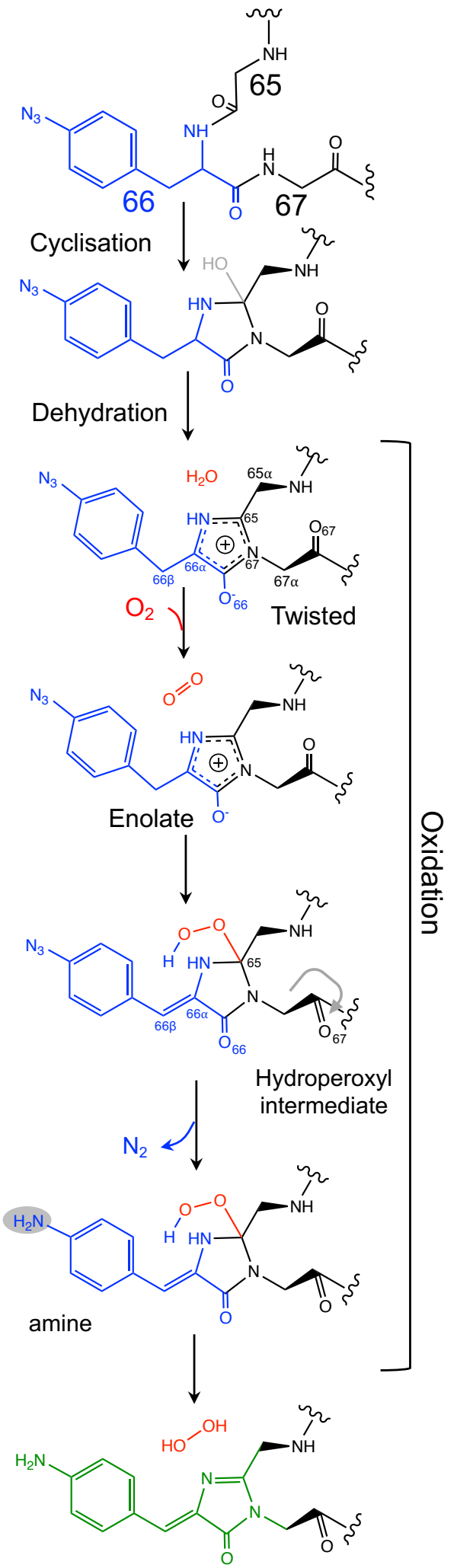

Figure 7. Proposed mechanism for the chromophore maturation, including details of the final oxidation step. 


\section{Discussion}

Photo-controllable FPs have become an important tool in modern super-resolution cell imaging ${ }^{38}$. The use of phenyl azide chemistry to control fluorescence, here and in green ${ }^{29,30}$ and red ${ }^{31}$ fluorescent proteins, provides a simple and general common mechanism to implement photocontrol across a broad range of the FP colour palette. Indeed, the use of a ncAA in conjunction with a reprogrammed amber stop codon allows secondary control: production of the fusion protein constructs with and without the FP adjunct ${ }^{39}$. While the demonstration of the conversion of an essentially colourless immature fluorescent protein to an active form through phenylazide photodecaging confirms the approach as a means to photo-control FPs, insights into the chromophore maturation process are arguably the most important aspect of this work. The one facet our data does confirm is that dehydration occurs before oxidation (Figure 2 and S3), the order of which has been debated in the past ${ }^{4}$. Based on our work, we propose a maturation route in Figure 7.

The crystal structure of the im-Venus ${ }^{66 \mathrm{azF}}$ provides evidence for the nature of an intermediate prior to the final oxidation step: the I ring in the enolate form with the $\mathrm{C}_{66 \alpha}$ and $\mathrm{C}_{66 \beta}$ forming a single bond (Figure $3 a$ ). The long $\mathrm{C}_{66}-\mathrm{O}_{66}$ bond is indicative of the enolate whose negative charge is stabilised by critical chromophore maturation residue, Arg96 (Figure 2a) ${ }^{14,18,40}$. The short $\mathrm{C}_{66}-\mathrm{N}_{67}$ bond (Figure 3a) indicates a $\mathrm{C}=\mathrm{N}$ bond that would put a positive charge on the nitrogen but the overall bond lengths of the I ring mean a more delocalised system. We don't preclude a positive charge on the $\mathrm{N}_{66}$; our structure indicates there would be no charge clash with Arg96 as suggested previously ${ }^{16}$.

The crystal structure also provides evidence of the location of $\mathrm{O}_{2}$. Two relative positions with respect to the chromophore plane have been proposed: on the Arg96 ${ }^{16}$ or the Glu222 face ${ }^{17}$. Here we show that molecular oxygen is placed on the Glu222 face, directly above the I ring (Figure 2). Arg96 has been suggested as the oxygen activator through the positively charged side-chain ${ }^{16}$ but this unlikely to be the case and may instead play a role in stabilising the enolate form of the I ring. 
The twisted Gly67 configuration is clearly observed in the crystal structure and differs from the canonical position normally present in FPs (Figure 2). Glycine has a less restricted $\psi$ angle range. In Venus, the Gly67 $\psi$ dihedral angle is $-23^{\circ}$ compared to $167^{\circ}$ in im-Venus ${ }^{66 a z F}$, with twisted conformation energetically less favourable (by 7 $\mathrm{kcal} / \mathrm{mol}$ ) when $\mathrm{O}_{2}$ is present and also hinders access of oxygen to the I ring (Figure 2). The twisted conformation could be a legacy of the cyclisation reaction whereby the nucleophilic attack of $\mathrm{N}_{67}$ on $\mathrm{C}_{65}$ will require rotation of the $\mathrm{Gly} 67 \psi$ angle leading to its observed placement in im-Venus ${ }^{66 a z F}$. The configuration could be stabilised by the $\mathrm{O}_{67} \mathrm{H}$-bonding with the $\mathrm{C}_{65}$ hydroxyl group (observed by Getzoff and colleagues ${ }^{15}$ ) before condensation. The simulation data reveal that water can stabilise the twisted conformation over the canonical form (Figure 5a), thus suggesting that a hydrated form precedes the oxygen bound step. It is interesting to speculate that the origin of the water molecule may be the product of the cyclisation/dehydration reaction that precedes oxidation. Indeed, while our crystal structure of im-Venus ${ }^{66 a z F}$ strongly indicates $\mathrm{O}_{2}$ is present, we cannot rule out that a minority population of the structures has a water molecule present in the same position.

If a water molecule originally stabilised the twisted Gly67 conformation, this suggests that $\mathrm{O}_{2}$ needs to access the protein core for the final oxidation step to take place. We suggest that His 148 plays a key role in this process. His 148 is dynamic ${ }^{41}$ and has been observed in both the "open" and "closed" conformations with the former not normally reported in the crystal structure as it is a minor component when observed (for example Arpino et al ${ }^{42}$, Reddington et al ${ }^{30}$ and Brejc et al ${ }^{43}$ ); the closed conformation is the major form observed in mature FPs as His148 in this configuration $\mathrm{H}$-bonds to the chromophore and plays a critical role in function 1,9,44,45. Im-Venus ${ }^{66 a z F}$ almost exclusively exists in the open conformation (Figure 2a and S3) that generates a channel through to the chromophore (Figure 6b-c). Such a tunnel at a similar position has been observed previously for GFP-like proteins ${ }^{46,47}$. Thus, His148 may acts as a "gatekeeper" residue, so determining access to the chromophore as well as its functional role ${ }^{41}$. Given that oxidation is the rate-limiting step in maturation ${ }^{4}$, it is interesting to speculate that the exchange rate between the two His148 conformations may play a role in defining this rate. 
Simulations predict that the next dominant form is hydroperoxyl intermediate attached to $C_{65}$ and not $C_{66 \alpha}$, as suggested by others ${ }^{16}$. The argument against attachment to $\mathrm{C}_{66 \alpha}$ as the intermediate comes from the observed spectra data (Figure 2b), whereby the dominant 350-360 nm peaks for the intermediate suggests some extension of the conjugated double bond system (here proposed to be from the phenyl azide to $\mathrm{C}_{66 \alpha}-\mathrm{C}_{66 \beta}$; Figure 5). The formation of a hydroperoxyl intermediate at $\mathrm{C}_{66 \alpha}$ would prevent the formation of the double bond with $\mathrm{C}_{66 \beta}$. The electron lone pair on the $\mathrm{C}_{65}$ hydroperoxyl moiety also aligns perfectly with the two $\mathrm{C}-\mathrm{N}$ bonds that, in turn, helps stabilise the five-membered I ring. We propose that the nearby Glu222 plays a vital role acting as a general acid/base during the formation of the hydroperoxyl intermediate through the first abstraction and then the donation of a proton to $\mathrm{N}_{66}$ (Figure S5). The importance of Glu222 to maturation has been observed previously, with the E222Q mutation in EGFP considerably slowing maturation ${ }^{18,40}$. During this process a peroxyanion is formed, which abstracts the proton from the activated $\mathrm{C}_{66 \beta}$ to form hydroperoxyl intermediate (Figure 4 and S5). Thus, the formation of the $\mathrm{C}_{66 \alpha}=\mathrm{C}_{66 \beta}$ double bond occurs before the generation of $\mathrm{H}_{2} \mathrm{O}_{2}$ and not concurrently (Figure 7).

The final and rate-limiting step in the process is the formation of the fully conjugated fluorescent chromophore. In our system, we believe this is a UV induced phase that happens in two steps due to the presence of the azido group: 1. Conversion of the phenyl azide to the phenylamine; 2 . Loss of the hydroperoxyl group so generating a fully conjugated system. In our proposed model, we suggest that the reduction of the azide to an amine occurs first (Figure 7). This is based on the predicted absorbance of each species (Figure S6a) and on the expectation that azide conversion to strongly donating amine group would significantly help with loss of the hydroperoxyl moiety (see Figure S6b for mechanistic details). Furthermore, the departure of the $\mathrm{OOH}$ group needed for the conversion of hydroperoxyl intermediate to the final maturated chromophore is expected to be greatly facilitated when azide, a mild acceptor (Hammett parameter $\left.\sigma_{p}=+0.08\right)$, is changed to $\mathrm{NH}_{2}$, a strong donor $\left(\sigma_{\mathrm{p}}=-\right.$ 0.66). In the azide-substituted peroxide, the lone pair of $\mathrm{N}_{66}$ is not able to fully assist in the departure of the $\mathrm{OOH}$ group as its electron density is partially delocalized in the other direction, towards the azide. This stereo-electronic tug-of-war is removed 
once the amine is formed. As the $\mathrm{NH}_{2}$ group is a powerful donor, the electron density is no longer shifted from $\mathrm{N}_{66}$ to the aryl ring; the lone pair on $\mathrm{N}_{66}$ is now free to stabilize the transition state for heterolytic $\mathrm{C}$... OOH bond scission. A fully conjugated chromophore with the azide group attached has a predicted $\lambda_{\max }$ of $451 \mathrm{~nm}$, whereas the amine version of hydroperoxyl intermediate is $367 \mathrm{~nm}$. Given the observed absorbance time course in figure 2 goes from a mixed species with two peaks between $340-360 \mathrm{~nm}$ to a single species at $360 \mathrm{~nm}$ that directly converts to the single species at $440 \mathrm{~nm}$, the logical progression of the predicted spectra are 360 $\mathrm{nm}$ (phenyl azide/hydroperoxyl form) to $367 \mathrm{~nm}$ (phenylamine/hydroperoxyl forms) to $444 \mathrm{~nm}$ (mature amine chromophore).

\section{Conclusion}

For the first time, our X-ray structure of im-Venus ${ }^{66 \mathrm{azF}}$ provides an experimental insight into the role played by oxygen in fluorescent protein chromophore maturation. The fortuitous trapping of oxygen between Tyr203 and an alternative conformation of the strictly conserved Gly67 before photo-decaging of azF66 was critical. From our structure and subsequent modelling, the cyclisation-dehydration-oxidation model mechanism is the likely route to chromophore maturation but with new insights concerning the nature of the enolate intermediate together with mechanistic details of the $\mathrm{O}_{2}$-dependent steps. These new insights not only improve our fundamental understanding of chromophore maturation but may help aid our ability to generate improved fluorescent protein for future applications. 


\section{Methods and materials}

\section{Engineering, production and structure of Venus ${ }^{66 \mathrm{azF}}$}

The generation of the mutant and subsequent recombinant production of Venus ${ }^{66 a z F}$ is outlined in the supporting information and based on a previously published procedure ${ }^{47}$. Protein production and purification was carried out in the dark to prevent photolysis of azF. Crystallisation, diffraction data collection and structure determination of im-Venus ${ }^{66 a z F}$ was performed as outlined in the supporting information. A long, spiny crystal of im-Venus ${ }^{66 a z F}$ was observed after a few days in the A12 condition of the PACT premierTM HT-96 screen (0.01 M Zinc chloride, $0.1 \mathrm{M}$ Sodium acetate, pH 5 and 20\% w/v PEG 6000). The structural statistics are provided in Supporting Table 2.

\section{Absorbance and fluorescence spectroscopy}

Absorbance spectra were recorded using a Cary 60 spectrophotometer (Agilent) in a $1 \mathrm{~cm}$ pathlength quartz cuvette. The molar absorbance coefficients were calculated by recording absorbance spectra of Venus samples with a known concentration (5$10 \mu \mathrm{M}$ ) and then extrapolated to $1 \mathrm{M}$ using the Beer-Lambert law. Emission spectra were recorded using a Cary Eclipse fluorimeter (Varian) using a $5 \mathrm{~mm} \times 5 \mathrm{~mm}$ QS quartz cuvette. Protein concentration of $0.5 \mu \mathrm{M}$ for Venus ${ }^{\mathrm{WT}}$ or $10 \mu \mathrm{M}$ for Venus ${ }^{66 a z F}$ was used with a fixed scan rate of $120 \mathrm{~nm} / \mathrm{min}$ with a $5 \mathrm{~nm}$ slit width. The excitation wavelength for Venus ${ }^{\mathrm{WT}}$ and Venus ${ }^{66 a z F}$ were determined from their absorbance spectrum. Bacterial live cell imaging was performed as outlined in the Supporting Information.

\section{Photolysis of Venus ${ }^{66 a z F}$}

Photolysis experiments were carried out using a UVM-57 Handheld UV lamp (6 W; $302 \mathrm{~nm}$ UV, UVP Cambridge, UK) and $1 \mathrm{~cm}$ pathlength quartz cuvette (Hellma), essentially as described previously ${ }^{29,46}$. A maximum of $500 \mu \mathrm{l}$ of protein sample (10 $\mu \mathrm{M})$ was pipetted into a cuvette and exposed to the UV $(302 \mathrm{~nm})$ for the indicated periods of time at a distance of $1 \mathrm{~cm}$. The absorbance spectra and emission spectra were recorded immediately afterwards, as described above.

\section{QM/MM Simulations}

Coordinates of heavy atoms of the im-Venus ${ }^{66 a z F-}$ crystal structure were used to construct a full atom three-dimensional model system. Previous examples in quantum mechanics/molecular mechanics (QM/MM) simulations of the mechanism of chromophore maturation included the wild-type GFP ${ }^{17}$, its Gly65-Gly66-Gly67 mutant ${ }^{48}$, and the recent modeling of Dreiklang ${ }^{49}$, a photoswitchable protein with the chromophore formed from the same amino acid residues Gly65, Tyr66, Gly67, as in Venus. Hydrogen atoms were added manually using molecular mechanics tools; the side chains of Arg and Lys were assumed as positively charged, the side chains of Glu and Asp as negatively charged. The model protein molecule was fully surrounded by explicit water molecules.

Structures of possible intermediates in the maturation reaction were optimized in QM/MM calculations. A large fraction of the chromophore-containing pocket was assigned to the QM-part. The Gly65-azF66-Gly67 fragment of the immature chromophore (CRO), the side chains from Arg96, Tyr203, Ser205, Glu222 and 4 water molecules were included. This initial composition was considered to model structures without the oxygen molecule. In majority of calculations, the $\mathrm{O}_{2}$ species 
was inserted to the cavity near CRO. Calculations of energies and energy gradients in QM were carried out using Kohn-Sham DFT with the PBE0 functional ${ }^{50}$ and the cc-pVDZ basis set. The AMBER force field was used in MM. The NWChem software package ${ }^{51}$ was applied to scan fragments of potential energy surface. To model the system in the triplet electronic state, the unrestricted DFT approach was used. Vertical excitation energies at selected points on the ground state potential energy surface were computed using the extended multiconfigurational quasi-degenerate perturbation theory in the second order (XMCQDPT2) ${ }^{52}$ the protocol that we verified earlier and used extensively in studies of the photoreceptor proteins ${ }^{53}$. Here, the perturbation theory calculations were based on the complete active space selfconsistent field (CASSCF) wavefunctions obtained by distributing 16 electrons over 12 orbitals and using density averaging over 15 states. To perform these calculations using the Firefly quantum chemistry package ${ }^{54}$, large molecular clusters including the QM parts of the system were selected. Natural Bond Orbital (NBO) analysis was used to evaluate stereoelectronic interactions ${ }^{55,56}$. Geometry optimizations for NBO evaluations were performed with $\left(\mathrm{SMD}^{57}=\right.$ water) for solvation corrections and the unrestricted wB97X DFT functional ${ }^{58}$ (with an integration grid of pruned 175,974 for first-row atoms and 250,974 for atoms in the second and later rows) with the 6$311++G(2 d, p)$ basis set for all atoms. Grimme's D2 version for empirical dispersion 59,60 was also included. Natural Bond Orbital (NBO) analyses were performed with NBO6 linked to Gaussian 16. They were used to gauge the magnitude of the hyperconjugative interactions in the presented systems. 


\section{Acknowledgements}

We would like to thank the staff at the Diamond Light Source (Harwell, UK) for the supply of facilities and beam time, especially Beamline 103 and 104 staff, under beamtime code $m \times 18812$. We thank BBSRC (BB/H003746/1 and BB/M000249/1), EPSRC (EP/J015318/1). H.S.A. was supported by the Higher Committee for Education Development in Iraq. We would like to thank the Protein Technology Hub, School of Biosciences, Cardiff University for use of facilities. B.L.G. and I.V.P. thank the Russian Science Foundation (project 17-13-01051) for support of the modelling part of this work. The calculations were carried out using the equipment of the shared research facilities of HPC computing resources at Lomonosov Moscow State University. The use of supercomputer resources of the Joint Supercomputer Center of the Russian Academy of Sciences is also acknowledged.

\section{Author contributions}

All authors contributed to the writing of the paper and analysing data. HSA. undertook structural biology and functional characterisation the Venus proteins; $\mathrm{TH}$ helped prepare mutant proteins. PJR collected structural data and helped with structure determination and refinement. BLG, IVP and AVN. developed computational models and carried out computer simulations. IA and GPG provided analysis of stereoelectronic factors involved in the formation and reactivity of hydroperoxide intermediates. CB analysed data. DDJ conceived and directed the project, and analysed data. 


\section{References}

1. Tsien, R.Y. The green fluorescent protein. Annual review of biochemistry 67, 509-44 (1998).

2. Zimmer, M. GFP: from jellyfish to the Nobel prize and beyond. Chem Soc Rev 38, 2823-32 (2009).

3. Pakhomov, A.A. \& Martynov, V.I. GFP family: structural insights into spectral tuning. Chemistry \& biology 15, 755-64 (2008).

4. Craggs, T.D. Green fluorescent protein: structure, folding and chromophore maturation. Chem Soc Rev 38, 2865-75 (2009).

5. Shaner, N.C., Steinbach, P.A. \& Tsien, R.Y. A guide to choosing fluorescent proteins. Nat Methods 2, 905-9 (2005).

6. Rodriguez, E.A. et al. The Growing and Glowing Toolbox of Fluorescent and Photoactive Proteins. Trends Biochem Sci 42, 111-129 (2017).

7. Nagai, T. et al. A variant of yellow fluorescent protein with fast and efficient maturation for cell-biological applications. Nat Biotechnol 20, 87-90 (2002).

8. Rekas, A., Alattia, J.R., Nagai, T., Miyawaki, A. \& Ikura, M. Crystal structure of venus, a yellow fluorescent protein with improved maturation and reduced environmental sensitivity. J Biol Chem 277, 50573-8 (2002).

9. Zimmer, M. Green fluorescent protein (GFP): applications, structure, and related photophysical behavior. Chem Rev 102, 759-81 (2002).

10. Wachter, R.M. Chromogenic cross-link formation in green fluorescent protein. Acc Chem Res 40, 120-7 (2007).

11. Reid, B.G. \& Flynn, G.C. Chromophore formation in green fluorescent protein. Biochemistry 36, 6786-91 (1997).

12. Pletneva, N.V. et al. Structural evidence for a dehydrated intermediate in green fluorescent protein chromophore biosynthesis. J Biol Chem 285, 1597884 (2010).

13. Rosenow, M.A., Huffman, H.A., Phail, M.E. \& Wachter, R.M. The crystal structure of the Y66L variant of green fluorescent protein supports a cyclization-oxidation-dehydration mechanism for chromophore maturation. Biochemistry 43, 4464-72 (2004).

14. Wood, T.I. et al. Defining the role of arginine 96 in green fluorescent protein fluorophore biosynthesis. Biochemistry 44, 16211-20 (2005).

15. Barondeau, D.P., Kassmann, C.J., Tainer, J.A. \& Getzoff, E.D. Understanding GFP chromophore biosynthesis: controlling backbone cyclization and modifying post-translational chemistry. Biochemistry 44, 1960-70 (2005).

16. Barondeau, D.P., Tainer, J.A. \& Getzoff, E.D. Structural evidence for an enolate intermediate in GFP fluorophore biosynthesis. J Am Chem Soc 128, 3166-8 (2006).

17. Grigorenko, B.L., Krylov, A.I. \& Nemukhin, A.V. Molecular Modeling Clarifies the Mechanism of Chromophore Maturation in the Green Fluorescent Protein. J Am Chem Soc 139, 10239-10249 (2017).

18. Sniegowski, J.A., Phail, M.E. \& Wachter, R.M. Maturation efficiency, trypsin sensitivity, and optical properties of Arg96, Glu222, and Gly67 variants of green fluorescent protein. Biochem Biophys Res Commun 332, 657-63 (2005). 
19. Shcherbakova, D.M. \& Verkhusha, V.V. Chromophore chemistry of fluorescent proteins controlled by light. Curr Opin Chem Biol 20, 60-8 (2014).

20. Adam, V., Berardozzi, R., Byrdin, M. \& Bourgeois, D. Phototransformable fluorescent proteins: Future challenges. Curr Opin Chem Biol 20, 92-102 (2014).

21. Lippincott-Schwartz, J. \& Patterson, G.H. Photoactivatable fluorescent proteins for diffraction-limited and super-resolution imaging. Trends in Cell Biology 19, 555-565 (2009).

22. Acharya, A. et al. Photoinduced Chemistry in Fluorescent Proteins: Curse or Blessing? Chem Rev 117, 758-795 (2017).

23. Henderson, J.N. et al. Structure and mechanism of the photoactivatable green fluorescent protein. J Am Chem Soc 131, 4176-7 (2009).

24. Ando, R., Hama, H., Yamamoto-Hino, M., Mizuno, H. \& Miyawaki, A. An optical marker based on the UV-induced green-to-red photoconversion of a fluorescent protein. Proc Natl Acad Sci U S A 99, 12651-6 (2002).

25. Brakemann, T. et al. A reversibly photoswitchable GFP-like protein with fluorescence excitation decoupled from switching. Nat Biotechnol 29, 942-7 (2011).

26. Ando, R., Mizuno, H. \& Miyawaki, A. Regulated fast nucleocytoplasmic shuttling observed by reversible protein highlighting. Science 306, 1370-3 (2004).

27. Delagrave, S., Hawtin, R.E., Silva, C.M., Yang, M.M. \& Youvan, D.C. Redshifted excitation mutants of the green fluorescent protein. Biotechnology ( $N$ Y) 13, 151-4 (1995).

28. Niu, W. \& Guo, J. Expanding the chemistry of fluorescent protein biosensors through genetic incorporation of unnatural amino acids. Mol Biosyst 9, 296170 (2013).

29. Reddington, S.C. et al. Directed evolution of GFP with non-natural amino acids identifies residues for augmenting and photoswitching fluorescence. Chem Sci 6, 1159-1166 (2015).

30. Reddington, S.C. et al. Different photochemical events of a genetically encoded phenyl azide define and modulate GFP fluorescence. Angew Chem Int Ed Engl 52, 5974-7 (2013).

31. Reddington, S.C. et al. Genetically encoded phenyl azide photochemistry drives positive and negative functional modulation of a red fluorescent protein. RSC Advances 5, 77734-77738 (2015).

32. Chin, J. et al. Addition of p-azido-L-phenylalanine to the genetic code of Escherichia coli. J Am Chem Soc 124, 9026-7 (2002).

33. Reddington, S., Watson, P., Rizkallah, P., Tippmann, E. \& Jones, D.D. Genetically encoding phenyl azide chemistry: new uses and ideas for classical biochemistry. Biochem Soc Trans 41, 1177-82 (2013).

34. Gomes, G.D.P., Vil, V., Terent'ev, A. \& Alabugin, I.V. Stereoelectronic source of the anomalous stability of bis-peroxides. Chem Sci 6, 6783-6791 (2015).

35. Juaristi, E., Dos Passos Gomes, G., Terent'ev, A.O., Notario, R. \& Alabugin, I.V. Stereoelectronic Interactions as a Probe for the Existence of the Intramolecular alpha-Effect. J Am Chem Soc 139, 10799-10813 (2017).

36. Vil, V.A. et al. Synthesis of unstrained Criegee intermediates: inverse a-effect and other protective stereoelectronic forces can stop Baeyer-Villiger rearrangement of $\mathrm{Y}$-hydroperoxy- $\mathrm{y}$-peroxylactones. Chemical Science 11, 5313-5322 (2020). 
37. Chovancova, E. et al. CAVER 3.0: a tool for the analysis of transport pathways in dynamic protein structures. PLoS Comput Biol 8, e1002708 (2012).

38. Shcherbakova, D.M., Sengupta, P., Lippincott-Schwartz, J. \& Verkhusha, V.V. Photocontrollable fluorescent proteins for superresolution imaging. Annu Rev Biophys 43, 303-29 (2014).

39. Arpino, J.A., Baldwin, A.J., McGarrity, A.R., Tippmann, E.M. \& Jones, D.D. Inframe amber stop codon replacement mutagenesis for the directed evolution of proteins containing non-canonical amino acids: identification of residues open to bio-orthogonal modification. PLoS One 10, e0127504 (2015).

40. Sniegowski, J.A., Lappe, J.W., Patel, H.N., Huffman, H.A. \& Wachter, R.M. Base catalysis of chromophore formation in Arg96 and Glu222 variants of green fluorescent protein. J Biol Chem 280, 26248-55 (2005).

41. Seifert, M.H. et al. Slow exchange in the chromophore of a green fluorescent protein variant. J Am Chem Soc 124, 7932-42 (2002).

42. Arpino, J.A., Rizkallah, P.J. \& Jones, D.D. Structural and dynamic changes associated with beneficial engineered single-amino-acid deletion mutations in enhanced green fluorescent protein. Acta Crystallogr D Biol Crystallogr 70, 2152-62 (2014).

43. Brejc, K. et al. Structural basis for dual excitation and photoisomerization of the Aequorea victoria green fluorescent protein. Proc Natl Acad Sci U S A 94, 2306-11 (1997).

44. Hsu, S.T., Blaser, G. \& Jackson, S.E. The folding, stability and conformational dynamics of beta-barrel fluorescent proteins. Chem Soc Rev 38, 2951-65 (2009).

45. Hartley, A.M. et al. Functional modulation and directed assembly of an enzyme through designed non-natural post-translation modification. Chem Sci 6, 3712-3717 (2015).

46. Hartley, A.M., Worthy, H.L., Reddington, S.C., Rizkallah, P.J. \& Jones, D.D. Molecular basis for functional switching of GFP by two disparate non-native post-translational modifications of a phenyl azide reaction handle. Chem Sci 7, 6484-6491 (2016).

47. Worthy, H.L. et al. Positive functional synergy of structurally integrated artificial protein dimers assembled by Click chemistry. Communications Chemistry 2, 83 (2019).

48. Grigorenko, B.L., Kots, E.D., Krylov, A.I. \& Nemukhin, A.V. Modeling of the glycine tripeptide cyclization in the Ser65Gly/Tyr66Gly mutant of green fluorescent protein. Mendeleev Communications 29, 187-189 (2019).

49. Grigorenko, B.L., Polyakov, I.V., Krylov, A.I. \& Nemukhin, A.V. Computational Modeling Reveals the Mechanism of Fluorescent State Recovery in the Reversibly Photoswitchable Protein Dreiklang. J Phys Chem B 123, 89018909 (2019).

50. Adamo, C. \& Barone, V. Toward reliable density functional methods without adjustable parameters: The PBE0 model. Journal of Chemical Physics 110, 6158-6170 (1999).

51. Apra, E. et al. NWChem: Past, present, and future. J Chem Phys 152, 184102 (2020).

52. Granovsky, A.A. Extended multi-configuration quasi-degenerate perturbation theory: The new approach to multi-state multi-reference perturbation theory. The Journal of Chemical Physics 134, 214113 (2011). 
53. Nemukhin, A.V., Grigorenko, B.L., Khrenova, M.G. \& Krylov, A.I.

Computational Challenges in Modeling of Representative Bioimaging

Proteins: GFP-Like Proteins, Flavoproteins, and Phytochromes. J Phys Chem B 123, 6133-6149 (2019).

54. Granovsky, A.A. Firefly version 8.

55. Weinhold, F., Landis, C.R. \& Glendening, E.D. What is NBO analysis and how is it useful? International Reviews in Physical Chemistry 35, 399-440 (2016).

56. Gomes, G.d.P. \& Alabugin, I. Stereoelectronic Effects: Analysis by Computational and Theoretical Methods. in Applied Theoretical Organic Chemistry 451-502 (WORLD SCIENTIFIC (EUROPE), 2017).

57. Marenich, A.V., Cramer, C.J. \& Truhlar, D.G. Universal solvation model based on solute electron density and on a continuum model of the solvent defined by the bulk dielectric constant and atomic surface tensions. J Phys Chem B 113, 6378-96 (2009).

58. Chai, J.D. \& Head-Gordon, M. Long-range corrected hybrid density functionals with damped atom-atom dispersion corrections. Phys Chem Chem Phys 10, 6615-20 (2008).

59. Grimme, S., Antony, J., Ehrlich, S. \& Krieg, H. A consistent and accurate ab initio parametrization of density functional dispersion correction (DFT-D) for the 94 elements H-Pu. J Chem Phys 132, 154104 (2010).

60. Grimme, S., Ehrlich, S. \& Goerigk, L. Effect of the damping function in dispersion corrected density functional theory. J Comput Chem 32, 1456-65 (2011). 


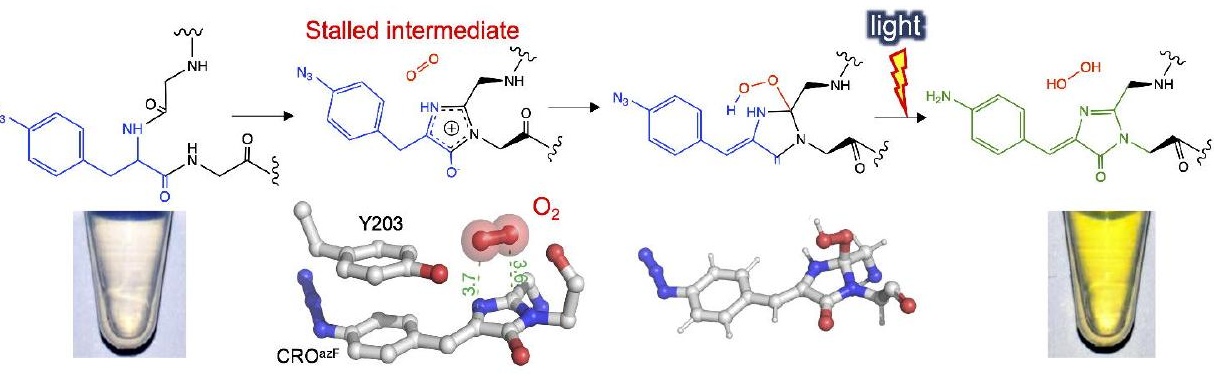

